\title{
Detecting Trace of Seam Carving for Forensic Analysis
}

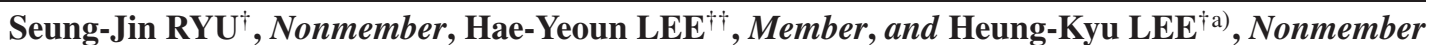

\begin{abstract}
SUMMARY Seam carving, which preserves semantically important image content during resizing process, has been actively researched in recent years. This paper proposes a novel forensic technique to detect the trace of seam carving. We exploit the energy bias and noise level of images under analysis to reliably unveil the evidence of seam carving. Furthermore, we design a detector investigating the relationship among neighboring pixels to estimate the inserted seams. Experimental results from a large set of test images indicates the superior performance of the proposed methods for both seam carving and seam insertion.

key words: image forensics, content-aware image resizing, seam carving, seam insertion
\end{abstract}

\section{Introduction}

The advances of imaging devices and computer software make the acquisition of high-quality digital images a natural form of human perception. At the same time, the very nature of digital images, which can be manipulated easily, brings into question many of the positive aspects associated with digital images. A constantly growing number of uncovered manipulations [1] is certainly only the tip of the iceberg.

Digital image forensics is the field of detecting modifications made to digital images. During the last decade, passive-blind [2] techniques have been developed to restore the lost trustworthiness of digital images. Generally, they require neither any access to the imaging device (a passive way) nor the knowledge about their inputs or intermediate results (a blind way) [3]. One of the key assumptions in passive-blind image forensics is that manipulating an image leaves inherent artifacts in the resulting image. Therefore, such artifacts may indicate a manipulation while examining the statistical properties of an image under investigation. For instance, many research works have revealed these inherent artifacts against manipulation: resampling [4]-[10], partial manipulation [11], [12], double quantization [13], [14], contrast enhancement [15], or sharpening [16].

Among the numerous post-processing techniques, resizing is one of the most frequently used. To detect the resizing artifacts, several detectors exploiting the periodic-

Manuscript received July 18, 2013.

Manuscript revised December 29, 2013.

†The authors are with the Department of Computer Science, Korea Advanced Institute of Science and Technology, Daejeon 305-701, Korea.

${ }^{\dagger \dagger}$ The author is with the School of Computer Science and Software Engineering, Kumoh National Institute of Technology, Gumi, Gyeongbuk 730-701, Republic of Korea.

a) E-mail: hklee@mmc.kaist.ac.kr

DOI: 10.1587/transinf.E97.D.1304 ity of interpolation through a resized image have been researched [4]-[10]. However, these detectors assume that the entire image is altered by a periodic resampling procedure. Hence, if part of the image is intentionally carved out or stretched while keeping the important content, by a manner of content-aware image resizing called seam carving [17], these detectors fail to detect the artifacts of resizing. Nevertheless, to the best of our knowledge, forensic detectors for the seam carving process have rarely been proposed. Therefore, in this paper, we propose a novel forensic detector by revealing the appropriate properties of the seam carving.

The rest of the paper is structured as follows. We first review prior works to reveal the trace of resizing in Sect. 2. Afterwards, the overview of a seam carving process is introduced in Sect. 3. Based on the analysis of the seam carving process, we propose appropriate features to detect the seam carved images in Sect. 4. Subsequently in Sect. 5, a seam insertion detector is proposed by tracking the trace of inserted seams in an image under investigation. Sect. 6 presents empirical evidence from a massive test setup before Sect. 7 concludes the paper.

\section{Review of the Major Resizing Detectors}

In this section, forensic detectors for either content independent or content-aware image resizing are briefly introduced. As mentioned in Sect. 1, image resizing is one of the most common forms of image manipulation. Since the traditional resizing process, which does not consider the content of the image, inherently incorporates resampling process, several detectors exploiting the periodicity of interpolation through a resampled image have been researched. Gallagher first uncovered periodicity in the second derivative signal of interpolated images [4]. The detector determined if the images had undergone resizing and the rate of interpolation. Mahdian and Saic analytically extended Gallaher's method [5]. They proved that the variance of the $n$-th order derivative of an interpolated signal has a periodicity equal to the sampling rate of the original signal if the given signal satisfies the stationary signal requirements. Aside from analyzing the $n$-th order derivative of the interpolated image, Popescu and Farid identified the correlation among neighboring pixels by a local linear predictor that adopts the expectation/maximization (EM) algorithm [6]. In [7], Kirchner analytically derived the relation between the derivative-based detector and Popescu's detector. From the analysis, he proposed an improved resampling detector. $\mathrm{He}$ 
also showed the specific periodicities that can be detected in a series of tailored row and column predictors instead of relying on a single predictor [8]. Feng et al. extracted 19 -features from the normalized energy density of second derivative images in the frequency domain, and then the feature vectors were applied to train and test a support vector machine (SVM) of resizing process [9]. To determine whether an investigated image is upscaled or downscaled, Pfennig and Kirchner combined Feng et al.'s approach with Popescu's linear predictor [10]. However, to the best of our knowledge, all the detectors still have difficulties in detecting the trace of content-aware image resizing, which intentionally carves out or stretches the part of the image without periodic resampling process.

A kind of content-aware image resizing, known as seam carving [17], keeps the important content of the image during the resizing process. Unfortunately, only several detectors for seam carving process were proposed. To detect the trace of seam carving, Lu and Wu attached side information called forensic hash to the image [18]. However, falsifiers can easily remove the forensic hash since it is attached on the header of an image file. Fillion and Sharma extracted many statistical features from seam carved images to detect the seam carving process [19]. Similarly, Sarkar et al. uncovered seam carving artifacts by extracting Markov process features [20]. Although these methods perform well, they should be improved by reflecting the important properties of the seam carving process. Therefore, the following sections introduces the details of seam carving process, and then the proposed method that considers the properties of the manipulation improved from the previous work [21] is presented.

\section{Seam Carving Process Overview}

Content-aware image resizing indicates that the important region of interest (ROI) is not affected (or minimally affected) by an image resizing process. Avidan and Shamir proposed a novel image retargeting method called seam carving [17]. A seam is an 8-connected path of pixels crossing the image from top to bottom, or from left to right. Therefore, a vertical seam for an $n \times m$ image $I$ is defined as Eq. (1) where $i$ and $x(i)$ represent row coordinates and the corresponding column coordinates, respectively. A horizontal seam is also defined similarly.

$$
\mathbf{s}^{\mathrm{V}}=\{(x(i), i)\}_{i=1}^{n}, \text { s.t. } \forall i,|x(i)-x(i-1)| \leqslant 1 .
$$

By successively removing unnoticeable seams which have as low energy as possible, or inserting additional seams right next to the selected unnoticeable seams, the important image content can be preserved during the resizing process. For this reason, the energy of each pixel is first measured by energy function $e$.

$$
e(\mathrm{I})=\left|\frac{\partial}{\partial x} \mathrm{I}\right|+\left|\frac{\partial}{\partial y} \mathrm{I}\right|
$$

From the energy function $e$, the energy of a vertical seam

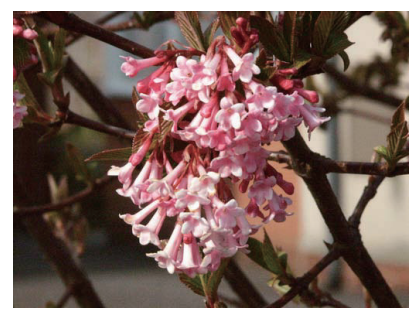

(a)

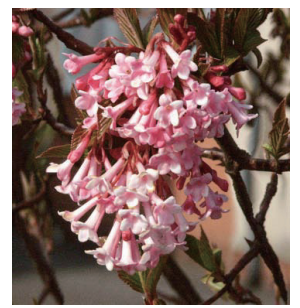

(c)

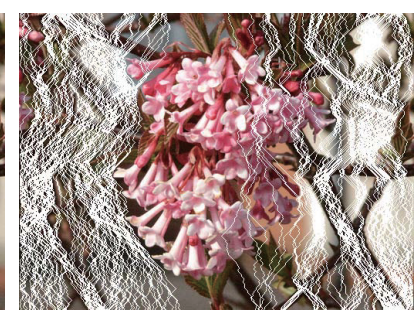

(b)

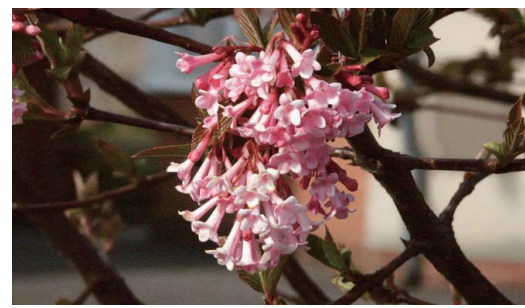

(d)
Fig. 1 Content-aware image resizing by seam carving: (a) an original image, (b) the original image with selected seams, (c) a seam carved image, and (d) a seam inserted image.

$\mathrm{E}(\mathbf{s})$ is as follows:

$$
\mathrm{E}(\mathbf{s})=\sum_{i=1}^{n} e(x(i), i), \text { s.t. } \forall i,|x(i)-x(i-1)| \leqslant 1 .
$$

Then, a vertical seam with the lowest energy path $s^{*}=$ $\min \mathrm{E}(\mathbf{s})$ is found by dynamic programming with the recurrence relation $M$ :

$$
\begin{aligned}
& M(i, j)=e(i, j)+ \\
& \quad \min (M(i-1, j-1), M(i, j-1), M(i+1, j-1)) .
\end{aligned}
$$

After constructing a cumulative minimum energy matrix $M$ for all possible seams, the lowest energy seam $s^{*}$ is found by backtracking from the minimum value of the last row in $M$. By iteratively eliminating the selected seam, which has the lowest energy, we can reduce the size of a given image without destroying significant image contents.

Enlarging an image is easily accomplished by extending the seam carving process. Instead of eliminating the selected vertical seam $s^{*}$, seam insertion process replaces pixels $s_{x(i), i}$ in the $s^{*}$ with $s_{x(i), i}^{l}$ and $s_{x(i), i}^{r}$, which are computed by averaging the $s_{x(i), i}$ with their left and right neighbors. Horizontal seams can be inserted in the similar way.

$$
\begin{aligned}
& s_{x(i), i}^{l}=\operatorname{round}\left(\frac{s_{x(i)-1, i}+s_{x(i), i}}{2}\right), \\
& s_{x(i), i}^{r}=\operatorname{round}\left(\frac{s_{x(i), i}+s_{x(i)+1, i}}{2}\right) .
\end{aligned}
$$

Figure 1 shows examples of seam carving and seam insertion. Even though we cut and put 30\% of the image through vertical seams, the important content of the image was not changed significantly.

\section{Seam Carving Detection}

By its very definition, seam carving process keeps an important image content by successively removing low energy seams. As a result, the energy distribution of seam 


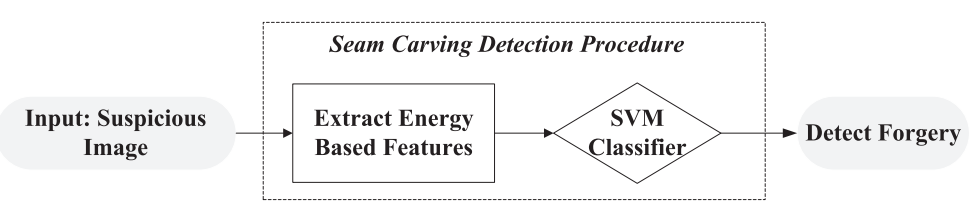

(a)

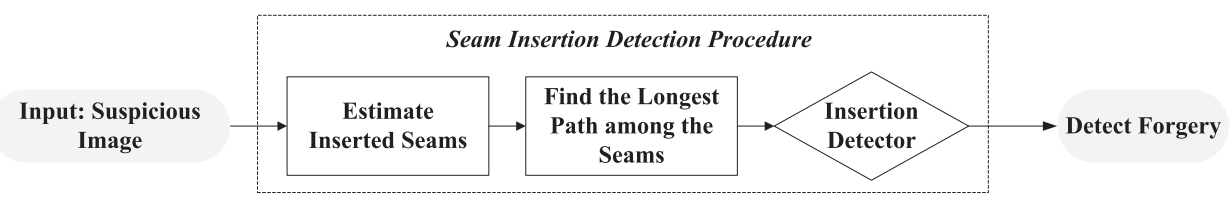

(b)

Fig. 2 The proposed detection procedure of (a) seam carving, and (b) seam insertion.

Table 1 Four features based on the average energy. The energy of a given image are computed by differentiating the image for both column and row directions.

\begin{tabular}{c|c}
\hline Feature & Description \\
\hline average column energy & $\frac{1}{m \times n} \sum_{i=1}^{m} \sum_{j=1}^{n}\left|\frac{\partial}{\partial x} \mathrm{I}(i, j)\right|$ \\
\hline average row energy & $\frac{1}{m \times n} \sum_{i=1}^{m} \sum_{j=1}^{n}\left|\frac{\partial}{\partial y} \mathrm{I}(i, j)\right|$ \\
\hline average energy & $\frac{1}{m \times n} \sum_{i=1}^{m} \sum_{j=1}^{n}\left(\left|\frac{\partial}{\partial x} \mathrm{I}(i, j)\right|+\left|\frac{\partial}{\partial y} \mathrm{I}(i, j)\right|\right)$ \\
\hline average energy difference & $\frac{1}{m \times n} \sum_{i=1}^{m} \sum_{j=1}^{n}\left(|| \frac{\partial}{\partial x} \mathrm{I}(i, j)|-| \frac{\partial}{\partial y} \mathrm{I}(i, j) \mid\right)$ \\
\hline
\end{tabular}

carved images is relatively higher than that of non-carved images [19]. Therefore, a set of features, which measures the energy bias of the image, is extracted to represent the characteristics of the seam carving process. We additionally analyze the energy of each seam. Furthermore, we adopt statistical noise measures. Seam carved images have much noise than non-carved images with high probability because the seam carving process mostly removes flat regions. As a consequence, three kinds of features extracted from statistically independent domains support to improve the detection performance of the seam carving process.

Afterwards, the feature vectors from the seam-carved and the non-carved images are applied to train and test Support Vector Machine (SVM) classifier [22]. Figure 2 (a) depicts a proposed detector for the seam carving process. In the following sub-sections, we first explain three kind of feature vectors by which local processing artifacts are unveiled, and then the SVM classifier is briefly described.

\subsection{Feature Extraction from Energy Distribution}

\subsubsection{Energy Features}

When the seams of an image are carved out, the average pixel energy for the image increases. Therefore, we measure the pixel energy by four statistical features as depicted in Table 1. Specifically, both the row and column direction average energy of each pixel are calculated from the investigated image since the seam carving process subsequently removes either row or column direction pixels. After that, the average energy feature including both the row and col- umn direction energy is computed. Moreover, we compute the difference between column and row direction energy. As mentioned above, seam carving process occurs through either column or row direction. Therefore, the subtraction between two directions reveals significant difference comparing to the subtraction from the non carved image.

\subsubsection{Seam Features}

After extracting features from the suspicious images, we measure the energy of each seam in the image. When an image is seam carved, the energy of remaining seams becomes higher than that of a non-carved image with high probability. From the above observation, we compute ten additional features in Table 2 using Eq. (4) for both row and column direction. After constructing a cumulative minimum energy matrix $M$ for all possible seams (for column and row directions), we compute five statistics values called min, max, mean, standard deviation, and the difference between maximum and minimum values for both directions. Similar with the energy features, we expect significant difference between seam energy values from seam carved images and those from non-carved images.

\subsubsection{Noise Features}

The seam carving process also affects the noise level of the manipulated images because the processing generally removes flat regions. From this observation, we measure four statistical noise features. To remove noise from the investigated image I, the image is filtered by Wiener filter with $5 \times 5$ window referred to as F. Finally, the noise $\mathrm{N}$ of the image is computed as follows.

$$
\mathrm{N}=\mathrm{I}-\mathrm{F}(\mathrm{I})
$$

From the computed noise, we measure mean, standard deviation, skewness, and kurtosis of the noise as depicted in Table 3. As a result, total 18 features are extracted.

\subsection{Support Vector Machine Classifier}

From the 18 features obtained by measuring the energy distribution, our purpose is to determine whether the image 
Table 210 features based on the vertical and horizontal seam energy by Eq. (4).

\begin{tabular}{|c|c|c|c|}
\hline Feature & Description & Feature & Description \\
\hline verticalseam $_{\max }$ & $\max _{i=1}^{m} M(i, n)$ & horizontalseam $_{\max }$ & $\max _{i=1}^{n} M(m, i)$ \\
\hline verticalseam $_{\min }$ & $\min _{i=1}^{m} M(i, n)$ & horizontalseam $_{\min }$ & $\min _{i=1}^{n} M(m, i)$ \\
\hline verticalseam ${ }_{\text {mean }}$ & $\frac{1}{m} \sum_{i=1}^{m} M(i, n)$ & horizontalseam $_{\text {mean }}$ & $\frac{1}{n} \sum_{i=1}^{n} M(m, i)$ \\
\hline verticalseam ${ }_{\text {std }}$ & $\sqrt{\frac{1}{m} \sum_{i=1}^{m}\left(\text { verticalseam }_{\text {mean }}-M(i, n)\right)^{2}}$ & horizontalseam $_{\text {std }}$ & $\sqrt{\frac{1}{n} \sum_{i=1}^{n}\left(\text { horisontalseam }_{\text {mean }}-M(m, i)\right)^{2}}$ \\
\hline verticalseam $_{\text {diff }}$ & verticalseam $_{\max }$ - verticalseam ${ }_{\min }$ & horizontalseam $_{\text {diff }}$ & horizontalseam $_{\max }-$ horizontalseam $_{\min }$ \\
\hline
\end{tabular}

Table 3 Four features based on the noise level. The noise of a given image is computed by Wiener filter with $5 \times 5$ window.

\begin{tabular}{c|c}
\hline Feature & Description \\
\hline mean & $\frac{1}{m \times n} \sum_{i=1}^{m} \sum_{j=1}^{n} \mathrm{~N}(i, j)$ \\
\hline standard deviation & $\sqrt{\frac{1}{m \times n} \sum_{i=1}^{m} \sum_{j=1}^{n}\left(\mathrm{~N}(i, j)-\mathrm{N}_{\text {mean }}\right)^{2}}$ \\
\hline skewness & $\frac{1}{m \times n} \sum_{i=1}^{m} \sum_{j=1}^{n}\left(\frac{\mathrm{N}(i, j)-\mathrm{N}_{\text {mean }}}{\mathrm{N}_{\text {std }}}\right)^{3}$ \\
\hline kurtosis & $\frac{1}{m \times n} \sum_{i=1}^{m} \sum_{j=1}^{n}\left(\frac{\mathrm{N}(i, j)-\mathrm{N}_{\text {mean }}}{\mathrm{N}_{\text {std }}}\right)^{4}$ \\
\hline
\end{tabular}

under investigation is seam-carved or not. To this end, we adopt the support vector machine (SVM) classifier which is a supervised learning method commonly used for classification [22]. In the training phase, the images for either class (seam-carved or non-carved) are represented by the feature vectors. The classifier then attempts to find an optimal linear decision surface called the maximum margin hyperplane by maximizing the geometric margin between the closest instances on either side. To further improve classification accuracy, the feature vectors are non-linearly projected into higher dimensional feature space by Radial Basis Function (RBF) kernel. After training the SVM classifier, a feature vector extracted from the image under investigation is classified into one of either side: seam-carved or non-carved.

\section{Seam Insertion Detection}

This section explains the proposed detector for seam insertion. As shown in Eq. (5), the seam insertion process introduces specific correlation among neighboring pixels through the selected seams. Using this correlation, Sarkar et al. proposed a localization method for inserted seams [20]. Without considering rounding, Eq. (7) can be derived from Eq. (5) if the examined pixels were generated by the seam insertion.

$$
\begin{aligned}
s_{x(i), i}^{l}-s_{x(i), i}^{r} & =\frac{s_{x(i)-1, i}+s_{x(i), i}}{2}-\frac{s_{x(i), i}+s_{x(i)+1, i}}{2} \\
& =\frac{s_{x(i)-1, i}-s_{x(i)+1, i}}{2} .
\end{aligned}
$$

Therefore, $s_{\text {diff }}$ in Eq. (8) should be 0 or 1 due to the rounding effect.

$$
\begin{aligned}
s_{\text {diff }} & =\left|\left(s_{x(i)-1, i}-s_{x(i)+1, i}\right)-2\left(s_{x(i), i}^{l}-s_{x(i), i}^{r}\right)\right| \\
& =0, \text { or } 1 .
\end{aligned}
$$

By calculating the $s_{\text {diff }}$ for every four horizontally sequential pixels in the image $I$, a binary map for the candidate location of the seam insertion $D$ is generated as below:

$$
D(i, j)=\left\{\begin{array}{l}
1, \text { if } s_{\text {diff }}=0, \text { or } 1 \\
0, \text { otherwise. }
\end{array}\right.
$$

We further notice that an estimated pixel $\hat{s}_{x(i), i}$ can be derived from Eq. (5) if the examined pixels were generated by the seam insertion.

$$
\hat{s}_{x(i), i}=\left(s_{x(i), i}^{l}+s_{x(i), i}^{r}\right)-2 \cdot\left(s_{x(i)-1, i}+s_{x(i)+1, i}\right) .
$$

Since $s_{x(i), i}^{l}$ and $s_{x(i), i}^{r}$ are calculated by averaging $s_{x(i), i}$ with their neighbors, we can derive the relation between the $\hat{s}_{x(i), i}$ and their neighbors as follows:

$$
\begin{aligned}
& s_{\text {corr }}^{l}=\left|\left(s_{x(i)-1, i}+\hat{s}_{x(i), i}\right)-2 \cdot s_{x(i), i}^{l}\right|=0, \text { or } 1 \\
& s_{\text {corr }}^{r}=\left|\left(\hat{s}_{x(i), i}+s_{x(i)+1, i}\right)-2 \cdot s_{x(i), i}^{r}\right|=0, \text { or } 1 .
\end{aligned}
$$

From Eq. (11), an additional map $C$ for the candidate location of the seam insertion is also generated.

$$
C(i, j)=\left\{\begin{array}{l}
1, \text { if }\left(s_{\text {corr }}^{l}=0, \text { or } 1\right) \wedge\left(s_{\text {corr }}^{r}=0, \text { or } 1\right) \\
0, \text { otherwise. }
\end{array}\right.
$$

By taking AND operation between two maps, $P=C \wedge D$, we generate a candidate map $P$. For a color image, we compute $P$ for each plane, and then apply AND operation among them to generate the only candidate map $P_{R G B}$. Finally, the decision for whether the image is enlarged by seam insertion or not is made by $P_{R G B}$. Specifically, if a vertical seam is inserted, there might exist a path with ' 1 ' from top to bottom. Hence, we compute the length of the longest path reaching to bottom end by dynamic programming with following recurrence relation $L$ :

$$
\begin{aligned}
& L(i, j)=P_{R G B}(i, j)+ \\
& \quad \max (L(i-1, j-1), L(i, j-1), L(i+1, j-1)) .
\end{aligned}
$$

If seams are inserted, the maximum value of the last row will be similar with the height $n$ of the image. On the contrary, if seams are not inserted, the maximum value of the last row will be significantly lower than $n$ caused by the absence of connected path. Thus, we decide the existence of the seam insertion procedure by Eq. (14) with a threshold weighting factor $t$. 


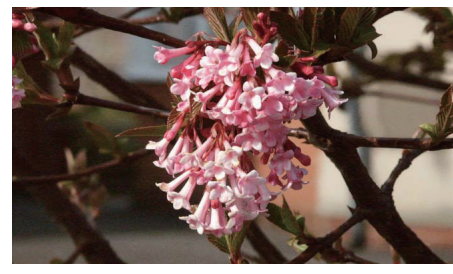

(a)

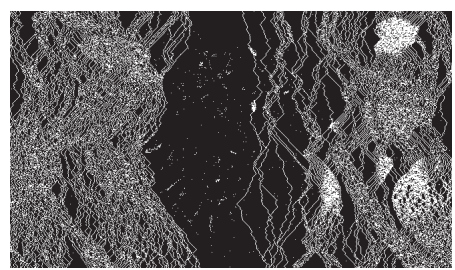

(c)

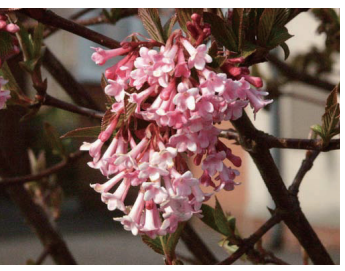

(b)

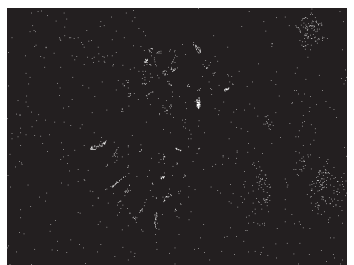

(d)

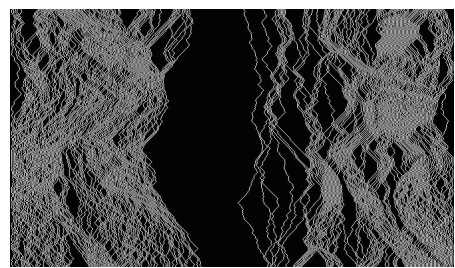

(e)

Fig. 3 Detection of inserted seams: (a) an enlarged image by seam insertion, (b) an original image, (c) detected seams from (a), (d) detected seams from (b), and (e) inserted seams into (a).

$$
\max _{i=1}^{m}(L(i, n)) \geqslant t \times n
$$

If at least one value satisfying Eq. (14) exists, we consider the examined image as a seam inserted image. Figure 2 (b) depicts the whole procedure of the proposed seam insertion detector. Simultaneously, Fig. 3 depicts detected seams by the proposed detector for both unchanged and seam inserted images. The result from our detector is displayed in Fig. 3 (c) and Fig. 3(d). We cannot find any seams in Fig. 3 (d) since Fig. 3 (d) is not manipulated by the seam insertion. On the contrary, Fig. 3 (c) coincides to a great extent with the ground truth map shown in Fig. 3 (e).

\section{Experimental Results}

This section reports results from an extensive series of content-aware image resizing detection experiments. The setup includes our method as detailed in Sect. 4 and Sect. 5 as well as two alternative detectors proposed by Sarkar et. al. [20] and Fillion and Sharma [19], respectively. With the detectors, 1,338 images from the UCID image set were used [23] to detect the artifacts of the resizing. More specifically, the images were reduced by seam carving from $10 \%$ to $50 \%$ in steps of $10 \%$. As a result, 6,690 carved images were generated. Also, the images were enlarged in a similar manner by seam insertion from $10 \%$ to $50 \%$ in steps of $10 \%$. Additionally, $1 \%$ enlarged images were generated to test the robustness of the seam insertion detectors. Thus, 8,028 images with inserted seams were generated. As a consequence,
Table 4 Detection results of $30 \%$ seam carving.

\begin{tabular}{cccc}
\hline \multirow{2}{*}{ Train } & \multicolumn{2}{c}{ Test[\%] } \\
\cline { 2 - 4 } & $\mathbf{3 0 \%}$ & non-carved \\
\cline { 2 - 4 } & $\mathbf{3 0 \%}$ & 82.51 & 17.49 \\
\hline \multirow{2}{*}{ non-carved } & 10.61 & 89.39 \\
\hline
\end{tabular}

1,338 original images, 6,690 seam carved images, and 8,028 seam inserted images were used in the experiments.

\subsection{Seam Carving Detection Results}

First, we classified whether images under investigation were seam carved or not. For this purpose, we trained LIBSVM classifier with Radial Basis Function (RBF) kernel of $r=0.125$ and $C=3$ [22]. The feature vector, which includes 18 statistics values explained in Sect. 4, was extracted from every seam-carved and non-carved images. Then, the half of feature vectors trained the SVM classifier. Finally, the remaining feature vectors were applied to test the performance of the detector. Table 4 depicts the confusion matrix of $30 \%$ seam carving and non-carved images by the proposed 18 features. To observe the detection performance under various environments, we varied the degree of seam carving from $10 \%$ to $50 \%$ in steps of $10 \%$. Moreover, the classifier was trained and tested with mixed set of seamcarved images, which include 268 randomly chosen images from $10 \%$ to $50 \%$ of seam carving, respectively. With the manipulated images, we tested the discrimination power of feature vectors as depicted in Table 5. It is worth to note that features based on seam property reveals best performance among the proposed features. However, from the result, we conclude that adopting every set of features demonstrates the best performance. Moreover, the performance of the proposed method as well as two alternative detectors was measured. Table 6 presents the detection performance of three detectors with different environments. Taking all tested images into account, the proposed method appears to be the most accurate approaches. Fillion and Sharma [19] proposed lots of features based on the energy histogram and higher order image statistics based on wavelet transformation. However, their features rarely unveil the property of each seam. Sarkar et al. [20], who consider seam carving as the change of inter-pixel correlation, adopt Shi-324 features. These features are also hard to measure the property of each seam. This is the reason why the performance of the proposed method is better than the others. The results also support that the proposed method is appropriate to detect the seam carving procedure unless the carved region is too small.

\subsection{Seam Insertion Detection Results}

The performance of the proposed method against seam insertion process was tested in this section. We not only estimated the inserted location of seams in a given image but also decided whether the image was forged or not. For this purpose, we computed a candidate map $P_{R G B}$ for each im- 
Table 5 Seam carving detection results, represented by accuracy, false positive rate (FPR), and false negative rate (FNR) values, by three feature vectors; Breakdown by feature vector and the amount of seam carving.

\begin{tabular}{|c|c|c|c|c|c|c|c|c|c|c|c|c|}
\hline \multirow[b]{2}{*}{ Reduction } & \multicolumn{4}{|c|}{ Accuracy [\%] } & \multicolumn{4}{|c|}{ False Positive Rate (FPR) [\%] } & \multicolumn{4}{|c|}{ False Negative Rate (FNR) [\%] } \\
\hline & Energy & Seam & Noise & All & Energy & Seam & Noise & All & Energy & Seam & Noise & All \\
\hline $10 \%$ & 52.69 & 68.36 & 53.44 & 71.60 & 47.98 & 24.96 & 31.54 & 19.43 & 46.64 & 38.27 & 61.58 & 37.37 \\
\hline $20 \%$ & 57.10 & 76.31 & 58.07 & 79.67 & 42.15 & 15.25 & 31.24 & 14.05 & 43.65 & 32.14 & 52.62 & 26.61 \\
\hline $30 \%$ & 62.18 & 82.66 & 62.10 & 85.95 & 31.99 & 11.36 & 31.24 & 10.61 & 43.65 & 23.32 & 44.54 & 17.49 \\
\hline $40 \%$ & 66.29 & 87.07 & 66.07 & 90.73 & 25.86 & 10.16 & 27.95 & 7.47 & 41.55 & 15.70 & 39.91 & 11.06 \\
\hline $\mathbf{5 0 \%}$ & 71.15 & 90.88 & 69.81 & 93.57 & 22.12 & 8.37 & 23.47 & 4.78 & 35.58 & 9.87 & 36.92 & 8.07 \\
\hline $\operatorname{mix}(10 \%-50 \%)$ & 63.78 & 86.33 & 65.05 & 88.95 & 32.88 & 9.12 & 39.46 & 5.83 & 39.55 & 18.21 & 30.45 & 16.27 \\
\hline
\end{tabular}

Table 6 Seam carving detection results, represented by accuracy, false positive rate (FPR), and false negative rate (FNR) values, by three seam carving detectors; Breakdown by detector and the amount of seam carving.

\begin{tabular}{|c|c|c|c|c|c|c|c|c|c|}
\hline \multirow[b]{2}{*}{ Reduction } & \multicolumn{3}{|c|}{ Accuracy [\%] } & \multicolumn{3}{|c|}{ False Positive Rate (FPR) [\%] } & \multicolumn{3}{|c|}{ False Negative Rate (FNR) [\%] } \\
\hline & Proposed & Sarkar et. al. & $\begin{array}{c}\text { Fillion and } \\
\text { Sharma }\end{array}$ & Proposed & Sarkar et. al. & $\begin{array}{c}\text { Fillion and } \\
\text { Sharma }\end{array}$ & Proposed & Sarkar et. al. & $\begin{array}{c}\text { Fillion and } \\
\text { Sharma }\end{array}$ \\
\hline $10 \%$ & 71.60 & 59.27 & 59.94 & 19.43 & 41.85 & 37.07 & 37.37 & 39.61 & 43.05 \\
\hline $20 \%$ & 79.67 & 66.07 & 63.75 & 14.05 & 36.77 & 35.87 & 26.61 & 31.09 & 36.62 \\
\hline $30 \%$ & 85.95 & 73.24 & 67.64 & 10.61 & 27.35 & 33.63 & 17.49 & 26.16 & 31.09 \\
\hline $40 \%$ & 90.73 & 79.97 & 69.96 & 7.47 & 21.82 & 31.99 & 11.06 & 18.24 & 28.10 \\
\hline $50 \%$ & 93.57 & 84.23 & 71.90 & 4.78 & 17.04 & 29.90 & 8.07 & 14.50 & 26.31 \\
\hline $\operatorname{mix}(10 \%-50 \%)$ & 88.95 & 71.63 & 61.11 & 5.83 & 11.96 & 17.04 & 16.27 & 44.78 & 60.75 \\
\hline
\end{tabular}

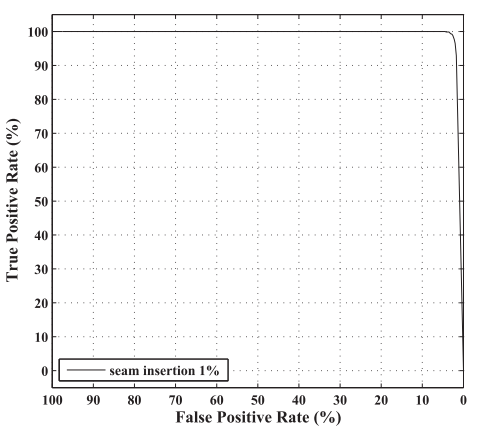

(a)

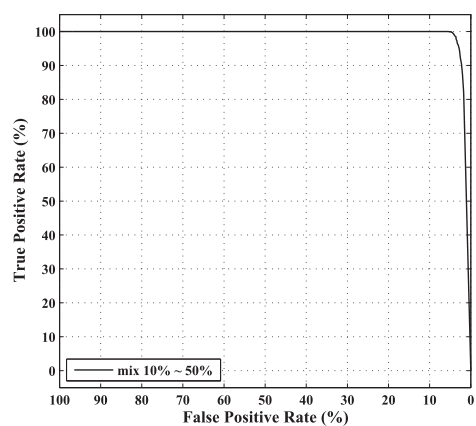

(b)

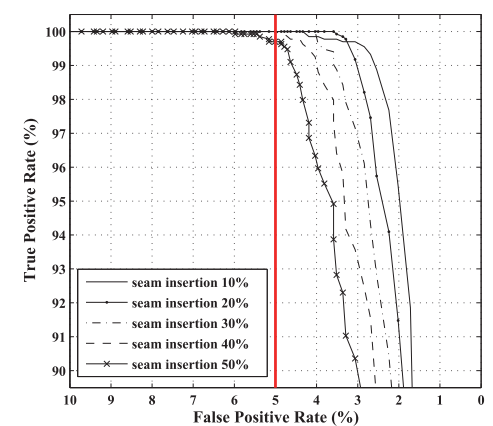

(c)

Fig. 4 Detection results of seam insertion with 1,338 UCID image set: (a) a ROC curve from original images and $1 \%$ seam inserted images, (b) a ROC curve from 1,338 original images and 1,340 images' set constructed with 268 randomly chosen images from $10 \%$ to $50 \%$ seam insertion, respectively, and (c) ROC curves by different amount of inserted seams.

age in the experimental set. Specifically, 8,028 maps to estimate the location of seam insertion were generated. As depicted in Fig. 3, we could notice that the proposed method is appropriate to localize the manipulated region. By using these maps, we further computed the recurrence relation $L$ in Eq. (13), and then drew several ROC curves by $t$ in Eq. (14). Figure 4 reports the corresponding ROC curves. Even if seams were rarely inserted (about 1\%), the proposed method was able to detect the trace of the insertion as shown in Fig. 4(a). Figure 4(b) shows the overall capability of detecting inserted seams from $10 \%$ to $50 \%$. At the same time, Fig. 4(c) exhibits the detailed performance of the proposed method with the different extent of the inserted seams. These experimental results support that the proposed method is enough to decide whether a given im- age is forged or not with low false positive error. Table 8 summarizes the performance of the proposed method by a different weighting factor $t$. From this table, we decided $t$ as 0.95 which satisfied both high accuracy and low false positive rate (FPR) error. With the selected weighting factor $t$, we compared the proposed method with other detectors. Table 7 depicts the detection accuracy of seam insertion by three different detectors. The proposed method generally yielded best performance except for insertion of $40 \%$ and $50 \%$. Even though Sarkar et. al.'s detector gave slight better performance with highly enlarged images, the overall performance of the proposed method outperformed prior arts. It is also worth noting that all but proposed method failed to reliably detect small amount of seam insertion (1\%) as reported in Table 7. 
Table 7 Seam insertion detection results, represented by accuracy, false positive rate (FPR), and false negative rate (FNR) values, by three seam insertion detectors; Breakdown by detector and the amount of seam insertion.

\begin{tabular}{|c|c|c|c|c|c|c|c|c|c|}
\hline \multirow[b]{2}{*}{ Insertion } & \multicolumn{3}{|c|}{ Accuracy $[\%]$} & \multicolumn{3}{|c|}{ False Positive Rate (FPR) [\%] } & \multicolumn{3}{|c|}{ False Negative Rate (FNR) [\%] } \\
\hline & Proposed & Sarkar et. al. & $\begin{array}{c}\text { Fillion and } \\
\text { Sharma }\end{array}$ & Proposed & Sarkar et. al. & $\begin{array}{c}\text { Fillion and } \\
\text { Sharma }\end{array}$ & Proposed & Sarkar et. al. & $\begin{array}{c}\text { Fillion and } \\
\text { Sharma }\end{array}$ \\
\hline $1 \%$ & 97.76 & 55.98 & 56.73 & 4.48 & 47.09 & 39.91 & 0.00 & 40.96 & 46.64 \\
\hline $10 \%$ & 97.76 & 89.24 & 61.51 & 4.48 & 10.31 & 48.73 & 0.00 & 11.21 & 28.25 \\
\hline $20 \%$ & 97.76 & 95.96 & 66.89 & 4.48 & 4.19 & 41.70 & 0.00 & 3.89 & 24.51 \\
\hline $30 \%$ & 97.76 & 97.31 & 74.36 & 4.48 & 1.94 & 32.14 & 0.00 & 3.44 & 19.13 \\
\hline $40 \%$ & 97.65 & 98.36 & 78.70 & 4.48 & 1.35 & 25.86 & 0.22 & 1.94 & 16.74 \\
\hline $\mathbf{5 0 \%}$ & 97.13 & 98.43 & 82.88 & 4.48 & 1.05 & 20.18 & 1.27 & 2.09 & 14.05 \\
\hline $\operatorname{mix}(10 \%-50 \%)$ & 97.61 & 90.89 & 66.18 & 4.48 & 2.39 & 19.43 & 0.30 & 15.82 & 48.21 \\
\hline
\end{tabular}

Table 8 Detection results of seam insertion by different weighting factor $t$ from 0.8 to 1 . The amount of inserted seams are varied from $1 \%$ to $50 \%$. 'mix' refers to mixed set of 1340 images, which is constructed with 268 randomly chosen images from $10 \%$ to $50 \%$ seam insertion. False Positive Rate (FPR) of unmanipulated images is also represented.

\begin{tabular}{c||c|c|c|c|c|c|c|c}
\hline $\boldsymbol{t}$ & FPR & $\mathbf{1 \%}$ & $\mathbf{1 0 \%}$ & $\mathbf{2 0 \%}$ & $\mathbf{3 0 \%}$ & $\mathbf{4 0 \%}$ & $\mathbf{5 0 \%}$ & $\mathbf{m i x}$ \\
\hline $\mathbf{1}$ & 1.72 & 92.90 & 91.85 & 87.07 & 81.02 & 77.28 & 73.32 & 82.11 \\
\hline $\mathbf{0 . 9 9}$ & 2.69 & 99.03 & 99.33 & 97.49 & 94.32 & 91.55 & 87.52 & 94.04 \\
\hline $\mathbf{0 . 9 5}$ & $\mathbf{4 . 4 8}$ & $\mathbf{1 0 0}$ & $\mathbf{1 0 0}$ & $\mathbf{1 0 0}$ & $\mathbf{1 0 0}$ & $\mathbf{9 9 . 7 8}$ & $\mathbf{9 8 . 7 3}$ & $\mathbf{9 9 . 7 0}$ \\
\hline $\mathbf{0 . 9}$ & 5.98 & 100 & 100 & 100 & 100 & 100 & 99.93 & 99.99 \\
\hline $\mathbf{0 . 8 5}$ & 8.30 & 100 & 100 & 100 & 100 & 100 & 100 & 100 \\
\hline $\mathbf{0 . 8}$ & 11.14 & 100 & 100 & 100 & 100 & 100 & 100 & 100 \\
\hline
\end{tabular}

\section{Conclusion}

The passive-blind forensic examination of visually convincing image manipulations faces a complex interplay of a variety of different processing steps. The empirical nature of digital images as well as their high dimensionality makes this problem analytically intractable in practice. This calls for the analysis of smaller sub-problems, where viable image models may at least exist [24]. In this paper, we have focused on the detection of content-aware image resizing by the seam carving process. To detect the trace of seam carving, we extracted the feature vector that measures the energy bias of a suspicious image. Afterwards, a pre-trained SVM classifier automatically determined whether the given image was manipulated. In case of seam insertion, we analyzed the relation among neighboring pixels, and then localized inserted seams. Experimental results based on the large set of images confirm the superior performance and the robustness of the presented approach under a variety of settings. As to the limitations, we note that most detectors are inherently incapable of localizing reduced regions by the seam carving process. The robustness against intentional distortions, such as compression, additive white Gaussian noise, or blurring will be considered as well.

\section{Acknowledgments}

This work was partially supported by Defense Acquisition Program Administration and Agency for Defense Development under the contract (UD060048AD).

\section{References}

[1] H. Farid, "Photo tampering throughout history," 2011.

[2] T.T. Ng, S.F. Chang, C.Y. Lin, and Q. Sun, "Passive-blind image forensics," Multimedia Security Technologies for Digital Rights, ed. W. Zeng, H. Yu, and C.Y. Lin, ch. 15, Academic Press, 2006.

[3] H.T. Sencar and N. Memon, eds., Digital Image Forensics: There is More to a Picture than Meets the Eye, Springer, 2013.

[4] A.C. Gallagher, "Detection of linear and cubic interpolation in jpeg compressed images," Proc. 2nd Canadian conference on Computer and Robot Vision, Washington, DC, USA, pp.65-72, 2005.

[5] B. Mahdian and S. Saic, "Blind authentication using periodic properties of interpolation," IEEE Trans. Information Forensics and Security, vol.3, no.3, pp.529-538, Sept. 2008.

[6] A.C. Popescu and H. Farid, "Exposing digital forgeries by detecting traces of re-sampling," IEEE Trans. Signal Process., vol.53, no.2, pp.758-767, 2005.

[7] M. Kirchner, "Fast and reliable resampling detection by spectral analysis of fixed linear predictor residue," Proc. 10th ACM workshop on Multimedia and security, MM\&Sec '08, pp.11-20, New York, NY, USA, 2008.

[8] M. Kirchner, "Linear row and column predictors for the analysis of resized images," MM\&Sec'10, Proc. 2010 ACM SIGMM Multimedia \& Security Workshop, pp.13-18, 2010.

[9] X. Feng, I.J. Cox, and G. Doerr, "An energy-based method for the forensic detection of re-sampled images," Proc. 2011 IEEE International Conference on Multimedia and Expo, ICME '11, Washington, DC, USA, pp.1-6, 2011.

[10] S. Pfennig and M. Kirchner, "Spectral methods to determine the exact scaling factor of resampled digital images," Communications Control and Signal Processing (ISCCSP), 2012 5th International Symposium on, pp.1-6, May 2012.

[11] X. Pan and S. Lyu, "Region duplication detection using image feature matching," IEEE Trans. Information Forensics and Security, vol.5, no.4, pp.857-867, 2010.

[12] S.J. Ryu, M.J. Lee, and H.K. Lee, "Detection of copy-rotate-move forgery using Zernike moments," Information Hiding, ed. R. Böhme, P. Fong, and R. Safavi-Naini, Lect. Notes Comput. Sci., vol.6387, Berlin, Heidelberg, pp.51-65, Springer-Verlag, 2010.

[13] A.C. Popescu and H. Farid, "Statistical tools for digital forensics," Information Hiding, ed. J. Fridrich, Lect. Notes Comput. Sci., vol.3200, Berlin, Heidelberg, pp.128-147, Springer-Verlag, 2004.

[14] Y.L. Chen and C.T. Hsu, "Detecting recompression of JPEG images via periodicity analysis of compression artifacts for tampering detection," IEEE Trans. Information Forensics and Security, vol.6, no.2, pp.396-406, 2011.

[15] M.C. Stamm and K.J.R. Liu, "Forensic detection of image manipulation using statistical intrinsic fingerprints," IEEE Trans. Information Forensics and Security, vol.5, no.3, pp.492-506, 2010.

[16] G. Cao, Y. Zhao, R. Ni, and A.C. Kot, "Unsharp masking sharpen- 
ing detection via overshoot artifacts analysis," IEEE Signal Process. Lett., vol.18, no.10, pp.603-606, 2011.

[17] S. Avidan and A. Shamir, "Seam carving for content-aware image resizing," ACM Trans. Graph., vol.26, no.3, July 2007.

[18] W. Lu and M. Wu, "Seam carving estimation using forensic hash," Proceedings of the thirteenth ACM multimedia workshop on Multimedia and security, MM\&Sec '11, New York, NY, USA, pp.9-14, 2011.

[19] C. Fillion and G. Sharma, "Detecting content adaptive scaling of images for forensic applications," Media Forensics and Security, SPIE Proc., p.75410, SPIE, 2010.

[20] A. Sarkar, L. Nataraj, and B.S. Manjunath, "Detection of seam carving and localization of seam insertions in digital images," Proc. 11th ACM workshop on Multimedia and security, MM\&\#38;Sec '09, New York, NY, USA, pp.107-116, 2009.

[21] S.J. Ryu, H.Y. Lee, and H.K. Lee, "Detection of content-aware image resizing using seam properties," Second International Conference on Engineering and Technology Innovation, 2012.

[22] C.C. Chang and C.J. Lin, "LIBSVM: A library for support vector machines," ACM Transactions on Intelligent Systems and Technology, vol.2, pp.27:1-27:27, 2011. Software available at http://www.csie.ntu.edu.tw/ cjlin/libsvm

[23] G. Schaefer and M. Stich, "UCID - an uncompressed colour image database," In Storage and Retrieval Methods and Applications for Multimedia 2004, vol.5307 of Proc. of SPIE, pp.472-480, 2004.

[24] R. Böhme, F. Freiling, T. Gloe, and M. Kirchner, "Multimedia forensics is not computer forensics," Computational Forensics, ed. Z.J. Geradts, K.Y. Franke, and C.J. Veenman, Lect. Notes Comput. Sci., vol.5718, pp.90-103, Berlin, Heidelberg, 2009.

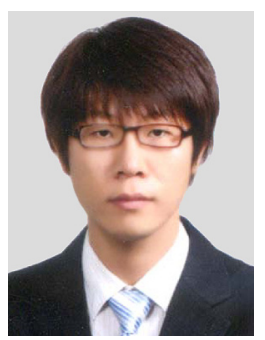

Seung-Jin Ryu received the B.S. degree in Computer Science from University of Seoul, Seoul, Republic of Korea, in 2007, and has been in the Integrated Masters Ph.D. Program in Computer Science from Korea Advanced Institute of Science and Technology (KAIST), Daejeon, Republic of Korea. He is currently pursuing the Ph.D. degree in Multimedia Computing Lab., Dept. of Computer Science, KAIST. His research interests include image and video forensics, data hiding, and steganography and

steganalysis.

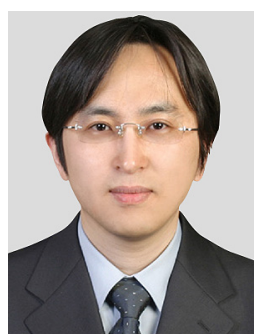

Hae-Yeoun Lee received his MS and $\mathrm{PhD}$ degrees in computer science from Korea Advanced Institute of Science and Technology (KAIST) in 1997 and 2006, respectively. From 2001 to 2006, he was with Satrec Initiative, Korea. From 2006 to 2007, he was a postdoctoral researcher at Weill Medical College, Cornell University, United States. He is now with Kumoh National Institute of Technology, Korea. His major interests are digital watermarking, digital forensics, image processing, remote sensing, and digital rights management.

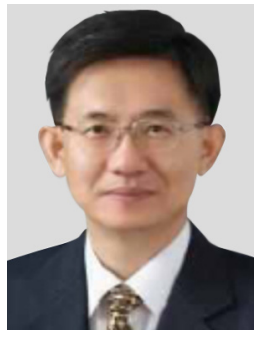

Heung-Kyu Lee received the BS degree in electronics engineering from the Seoul National University, Seoul, Korea, in 1978, and M.S., Ph. D. Degrees in computer science from the Korea Advanced Institute of Science and Technology (KAIST), in 1981, and 1984, respectively. Since 1986 he has been a professor of the Department of Computer Science, KAIST, Korea (hklee@mmc.kaist.ac.kr). His research team was selected as the Lab. Of Excellence (LOE), the National Research Lab (NRL), and the National Leading Research Lab (NLRL) by Korea Industry Technology Foundation in 2005 and National Research Foundation of Korea in 2007 and 2012, respectively. His major interests are information hiding and multimedia forensics. Prof. Lee is working with many government institutes and companies to develop the practical products in Korea. Since 2005, he received more than 20 research grants totaling over 6 million US dollars for projects on information hiding and multimedia forensics. He has authored/coauthored over 200 international papers. 